\title{
Acute pancreatitis as the debut of a pancreatic lesion
}

\section{Abstract}

To distinguish autoimmune pancreatitis (AIP), especially the focal type, from pancreatic cancer, is a great challenge for the clinician. Especially if it is the first episode of acute pancreatitis.

We present the case of a 45 -year-old patient who debuted with acute pancreatitis. A pancreatic mass was found within the etiological study. It was necessary to differentiate between a neoplasm and a focal autoimmune pancreatitis. Both pathologies have a diametrically different therapeutic approach.

Keywords: autoimmune pancreatitis, pancreatic focal lesion
Volume 4 Issue 4 - 2017

\author{
Paulina Nuñez,' Constanza Bay, ${ }^{2}$ Pamela \\ Yaquich,' Carrasco Gonzalo, ${ }^{3}$ Felipe Salem, ${ }^{4}$ \\ Emily Osse, ${ }^{4}$ Alberto Espino ${ }^{5}$ \\ 'Department of Gastroenterology, University of Chile, Chile \\ ${ }^{2}$ Department of Pediatrics, Pontifical Catholic University of \\ Chile, Chile \\ ${ }^{3}$ Department of Anatomy pathology, University of Chile, Chile \\ ${ }^{4}$ Medical student, University of Chile, Chile \\ ${ }^{5}$ Department of Gastroenterology, Pontifical Catholic University \\ of Chile, Chile
}

\section{Correspondence: Paulina Nuñez, Santiago, Department of Gastroenterology, University of Chile, Chile, Email paulinanunez@gmail.com \\ Received: November 05, 2017 | Published: November 27 2017}

Abbreviations: AIP, autoimmune pancreatitis; MRI, magnetic resonance imaging; CT, computerized tomography scan; EUS, endoscopic ultrasound

\section{Introduction}

The most frequent causes of acute pancreatitis are alcoholic, biliary, hypertriglyceridemia and drugs. ${ }^{1}$ However, it may also be the debut of a focal AIP or the presentation of a pancreatic neoplasia. Therefore, this etiologies should always be included in the differential diagnosis.

When we face a pancreatic lesion, it is necessary to consider the signs and symptoms, laboratory tests, images and the pathology. ${ }^{1,2}$ The clinical presentation as obstructive jaundice in patients with a pancreatic mass is common. Typical immunological abnormalities of autoimmune pancreatitis are high levels of serum gammaglobulin (IgG or IgG4). ${ }^{3}$

A focal lesion can be detected by computerized tomography scan (CT) or magnetic resonance imaging (MRI). According to clinical guidelines it is then necessary to perform an endosonography to differentiate the lesion by performing a fine needle puncture. The histopathological findings in AIP typically show infiltration of lymphocytes, IgG4-positivity and storiform fibrosis. ${ }^{4,5}$

These findings allow us to distinguish between a neoplasm that usually requires a surgical approach and an autoimmune pancreatitis that responds to steroid treatment.

\section{Case presentation}

45year-old man with no medical or surgical history, no alcohol or tobacco consumption, no known allergies or family medical history. With history of abdominal pain in the upper abdomen for about one year. Since one month ago referring with greater intensity in the left hypochondrium. The day of the consult pain intensifies up to $8 / 10$ VAS with irradiation to the back. It was not associated with nausea, vomiting or jaundice. Patient was evaluated at the emergency department where he was found hemodynamically stable, afebrile, without jaundice, with no signs of peritoneal irritation and with diminished bowel sounds. Initial laboratory tests: hematocrit $42 \%$, hemoglobin 14gr/dl, leukocytes 9000/uL, platelets 245000/uL, CRP $12 \mathrm{mg} / \mathrm{dl}$, AST 16IU/ml, (35) ALT 27IU/ml (50), ALP 65IU/ml (70), GGT 28IU/ml, total bilirubin 0.4mg/dl (1), Lipase 2744U/L, Amylase 204U/L. An abdominal CT scan was performed which showed peripancreatic edema and inflammatory changes of the peripancreatic adipose tissue (Figure 1). After initial study, mild acute pancreatitis was diagnosed. Biliary etiology was ruled out with an abdominal echotomography that showed a distended gallbladder with no stones, intra- and extra hepatic thin biliary tract. He had no drug or alcohol consumption and triglycerides were in normal range. Abdominal MRI showed a dilated pancreatic duct of $5 \mathrm{~mm}$, prominent pancreas and amputation of the pancreatic duct, without evidence of solid or cystic pancreatic lesion (Figure 2). Additional laboratory tests Ca19-9and serum IgG4 were within the normal ranges.

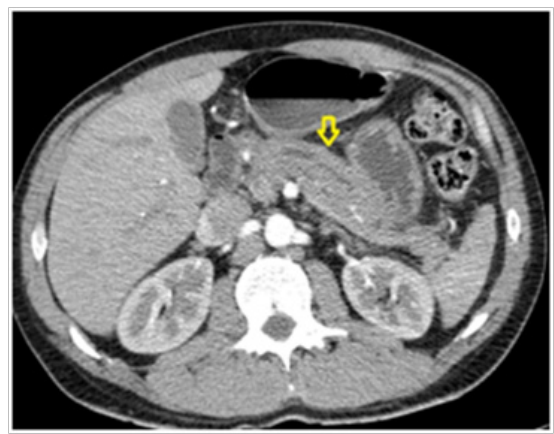

Figure I CT, peripancreatic edema. 


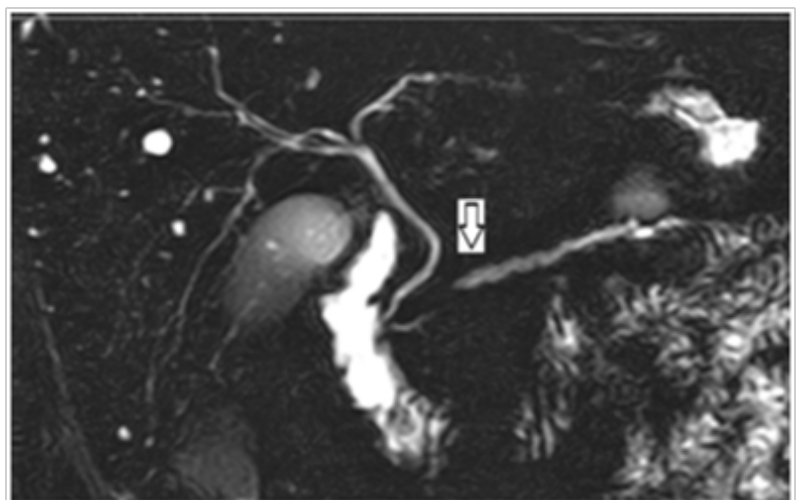

Figure 2 MRI cholangiography phase: amputation of the pancreatic duct.

Endosonography showed a heterogeneous and hypoechoic nodular lesion of $15 \mathrm{~mm}$ in the head of the pancreas. It was poorly delimited and had irregular edges. Ultrasound elastography revealed an indurated lesion and an area where the dilation of the pancreatic duct suddenly narrowed (Figure 3). Fine needle aspiration was performed and findings of the cytological and cell block study indicated an adenocarcinoma. The case was consulted with the surgical team and a pancreatoduodenectomy was performed without incidents. Biopsies demonstrated glandular atrophy and lymphocyte plasmocytic infiltrate (Figure 4). Immunotyping evidenced IgG4 presence in the cytoplasm of plasma cells, with an IgG4/IgG ratio greater than $40 \%$ (Figure 5). Finally, a focal autoimmune pancreatitis was diagnosed.

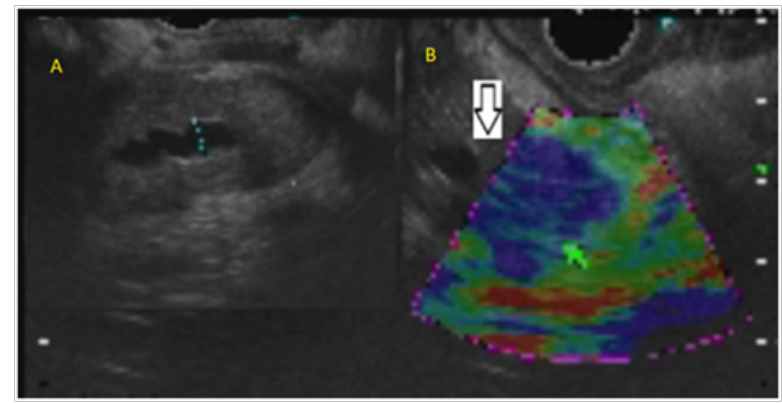

Figure $3 \mathrm{~A}$. dilation of the pancreatic duct suddenly narrows. B. indurated lesion.

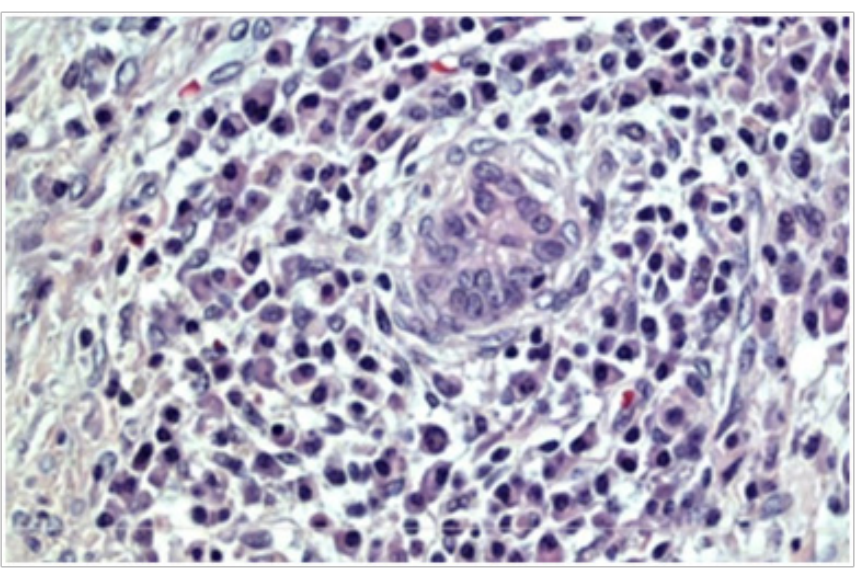

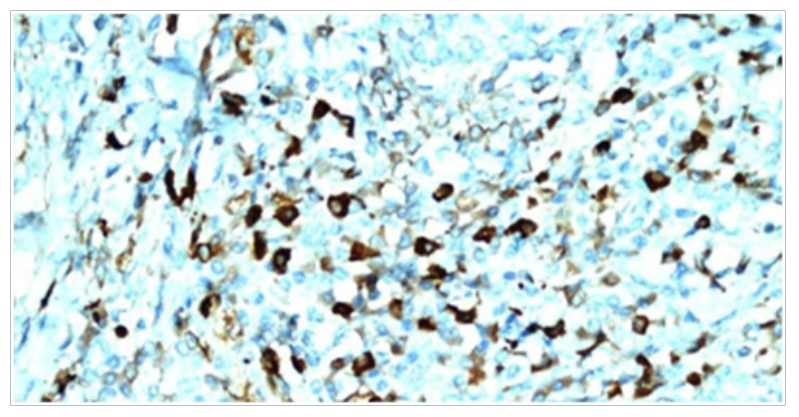

Figure 5 Immunotyping, $\lg G 4(+)$.

\section{Discussion}

This case illustrates the differential diagnosis of acute pancreatitis as a debut of a pancreatic lesion. AIP can be difficult to distinguish from pancreatic malignancy. The diagnosis of a pancreatic mass usually requires correlation of clinical, laboratory, imaging and pathology data.

Autoimmune pancreatitis has been considered a rare disease, but over time its diagnosis has increased. Two distinct subtypes of AIP have been established, type 1 and type 2 . Type 1 is also referred to as lymphoplasmacytic sclerosing pancreatitis (LPSP).It is the pancreatic manifestation of the systemic IgG4-related disease (IgG4-RD). However, laboratory does not always show elevated serum IgG4 levels. ${ }^{6}$ This form of the disease is more common in Asian countries. Type 2 AIP is also known as idiopathic duct-centric pancreatitis (IDCP).

The current diagnostic criteria rely on the use of imaging modalities such as computed tomography (CT), magnetic resonance cholangiopancreatography (MRCP), and endoscopic ultrasound (EUS). EUS may also demonstrate a focal solitary mass, which is typically visualized as a hypoechoic lesion, commonly located in the pancreatic head. EUS findings of the pancreatic parenchyma may also mimic typical chronic pancreatitis including the presence of hyperechoic foci, hyperechoic strands, and lobularity.

In addition to providing imaging hints to the diagnosis, EUS may confirm the diagnosis of AIP by EUS-guided biopsy. The most frequent findings are increased inflammatory lymphoplasmacytic infiltrate andstoriform fibrosis. This fibrosis has characteristic dense, wire like strands of fibrotic collagen deposition radiating outward from a central point. ${ }^{6}$ The presence of storiform fibrosis and obliterative phlebitis are more common in type 1 AIP, but they can also be observed in type 2 AIP.

Other elements include small aggregates of B cells, which may be distributed in germinal centers depending on the anatomic location. Tissue eosinophilia is usually present.

When the diagnosis is established, most patients respond to treatment with corticosteroids. Both retrospective ${ }^{8}$ and prospective ${ }^{9}$ studies have demonstrated the effectiveness of steroid therapy to initiate remission. Subsequent management includes observation or corticoid therapy at low doses. ${ }^{10}$ In non-responders there is experience with the use of rituximab.

Figure 4 lymphocytoplasmocytic infiltrate. 


\section{Acknowledgements}

None.

\section{Conflicts of interest}

Author declares that there is no conflict of interest.

\section{Patient consent form}

Patient's permission to publish this case report was obtained and documented in a patient consent form.

\section{References}

1. Webster GJ. Autoimmune pancreatitis - a riddle wrapped in an enigma. Dig Dis. 2016;34(5):532-539.

2. Chintanaboina J, Yang Z, Mathew A. Autoimmune pancreatitis: a diagnostic challenge for the clinician. South Med J. 2015;108(9):579-589.

3. Madhani K, Farrell JJ. Autoimmune pancreatitis an update on diagnosis and management. Gastroenterol Clin N Am. 2016;45(1):29-43.
4. Okun SD, Lewin DN. Non- neoplastic pancreatic lesions that may mimic malignancy. Semin Diagn Pathol. 2016;33(1):31-42.

5. Hart PA, Krishna SG, Okazaki K. Diagnosis and management of autoimmune pancreatitis. Curr Treat Options Gastroenterol. 2017;15(4):538-547.

6. Weindorf SC, Frederiksen JK. IgG4 related disease: A reminder for practicing pathologists. Arch Pathol Lab Med. 2017;141(11):1476-1483.

7. Fuji Lau L, Levy MJ. The role of endoscopic ultrasound in the diagnosis of autoimmune pancreatitis. Gastrointest Endosc Clin N Am. 2017;27(4):643-655.

8. Hart PA, Topazian MD, Witzig TE, et al. Treatment of relapsing autoimmune pancreatitis with immunomodulators and rituximab: The Mayo Clinic experience. Gut. 2013;62(11):1607-1615.

9. Sandanayake NS, Church NI, Chapman MH, et al. Presentation and management of post-treatment relapse in autoimmune pancreatitis/ immunoglobulin G4-associated cholangitis. Clin Gastroenterol Hepatol. 2009;7(10):1089-1096.

10. Sah RP, Chari ST. Recent developments in steroid-responsive pancreatitis (autoimmune pancreatitis). Curr Opin Gastroenterol. 2015;31(5):387-394. 